\title{
On the first Laplacian eigenvalue and the center of gravity of compact hypersurfaces
}

\author{
Alain R. Veeravalli
}

\begin{abstract}
For a closed hypersurface in a space form, this work provides some sharp upper bounds for its first positive Laplacian eigenvalue. These bounds are extrinsic as they rely on the mean curvatures and center(s) of gravity of the hypersurface.
\end{abstract}

Mathematics Subject Classification (2000). 53C40, 53C20, 53C42.

Keywords. Hypersurface, space form, center of gravity, Laplacian eigenvalue, mean curvatures.

\section{Notations, introduction and the result}

Let $\left(\mathbb{M}=\mathbb{M}_{n+1}(\kappa), g=\langle\cdot, \cdot\rangle\right)$ be a space form, that is a connected simply connected Riemannian manifold of dimension $n+1 \geq 2$, with constant sectional curvature $\kappa \in \mathbb{R}, d$ its Riemannian distance, $d \tilde{\nu}$ its volume element, $\widetilde{\nabla}$ its LeviCivita connection, $\widetilde{\nabla}^{2}$ its Hessian, $\widetilde{\Delta}=-$ trace $\widetilde{\nabla}^{2}$ its Laplacian, exp its exponential map and $U_{p} \mathbb{M}$ the unit sphere of the tangent space $T_{p} \mathbb{M}$ of $\mathbb{M}$ at a point $p$. If $M$ is a closed (compact without boundary) connected hypersurface of $\mathbb{M}$, we endow $M$ with the induced metric, also denoted by $\langle\cdot, \cdot\rangle$. The induced volume element, connection, Hessian and Laplacian are denoted by $d \nu, \nabla, \nabla^{2}$ and $\Delta$ respectively. We recall that its mean curvatures are the functions $\left(H_{i}\right)_{0 \leq i \leq n}$ defined by $\prod_{i=1}^{n}\left(1+X k_{i}\right)=\sum_{i=0}^{n}\left(\begin{array}{c}n \\ i\end{array}\right) H_{i} X^{i}$ where $\left(k_{i}\right)_{1 \leq i \leq n}$ are the principal curvatures of $M$. By the generalized Jordan theorem, $M$ is orientable and divides $\mathbb{M}$ into two connected components, one of which, say $\Omega$, is relatively compact and has $M$ as its oriented boundary. We introduce the function $\mathrm{sn}_{\kappa}$ solution of the differential equation $\ddot{y}+\kappa y=0$ with the initial conditions $(y(0), \dot{y}(0))=(0,1))$ and its primitive $h_{\kappa}$ which vanishes at 0 . A center of gravity of $M$ is a critical point of the smooth function $\mathcal{E}: \mathbb{M} \rightarrow \mathbb{R}: p \mapsto \int_{M} h_{\kappa} \circ d_{p} \cdot d \nu$. This definition differs slightly from the one commonly used. The introduction of $h_{\kappa}$ has an immediate utility: the distance function $d_{p}$ may be non smooth at some points but, thanks to $h_{\kappa}, \mathcal{E}$ is nevertheless smooth on the whole manifold $\mathbb{M}$. Center(s) of gravity has several applications: for example, we recall that it can be used to prove that any compact 
group of isometries in an Hadamard manifold has a fixed point. In this paper, we will use center(s) of gravity to provide some sharp upper bounds for the first positive Laplacian eigenvalue involving the mean curvatures of $M$. The method used here is to apply the min-max principle to a collection of appropriate functions and goes back to Bleecker and Weiner [2]. Their work, with the generalization given by Reilly [3], dealt with Euclidean submanifolds. The author was naturally led to expect similar results for space form submanifolds. This attempt has been possible by the introduction of center(s) of gravity. The proof will show that the function $h_{\kappa}$ will make possible the use of generalized Hsiung-Minkowski formulae. For sake of simplicity, this work concerns only hypersurfaces but for higher codimension, similar formulae can be derived. Our result is the following one (the case $\kappa=0$ is the one studied by the authors quoted above):

Theorem. Let $M$ be a closed connected hypersurface of $\mathbb{M}, \lambda_{1}$ the first positive eigenvalue of the Laplacian of $M$ and $c$ a center of gravity of $M$.

i) If $\kappa$ is non-negative, then we have the following inequalities:

$$
\begin{gathered}
\lambda_{1} \int_{M} \operatorname{sn}_{\kappa}^{2} \circ d_{c} \cdot d \nu \leq n \operatorname{Vol} M \\
\lambda_{1}\left(\int_{M} H_{i} \sin _{\kappa} \circ d_{c} \cdot d \nu\right)^{2} \leq n \operatorname{Vol} M \int_{M} H_{i+1}^{2} \cdot d \nu \\
\lambda_{1}(n+1)^{2}\left(\int_{\Omega} \sin _{\kappa} \circ d_{c} \cdot d \tilde{\nu}\right)^{2} \leq n \operatorname{Vol}^{2} M
\end{gathered}
$$

for any $i \in \llbracket 0, n-1 \rrbracket$ where Vol $M$ denotes the volume of $M$. Equality occurs in one of these three inequalities if and only if $M$ is a geodesic sphere centered at $c$. In this case, $\lambda_{1}=n / \mathrm{sn}_{\kappa}^{2} r, r$ being the radius of this geodesic sphere.

ii) If $\kappa$ is negative, then

$$
\lambda_{1} \int_{M} \operatorname{sn}_{\kappa}^{2} \circ d_{c} \cdot d \nu \leq n \operatorname{Vol} M-\kappa \int_{M} \operatorname{sn}_{\kappa}^{2} \circ d_{c} \cdot d \nu
$$

For negative $\kappa$, inequality (4) is non sharp unfortunately (see the final remark) and this means that the min-max principle has to be applied to "better" functions. Before going further, the problem of the existence of a center of gravity has to be studied: while for positive $\kappa$, two centers of gravity, at least, exist by the compactness of $\mathbb{M}$, the question for non-positive $\kappa$ is solved by the following result:

Proposition 1. The function $h_{\kappa} \circ d_{p}$ satisfies:

$$
\widetilde{\nabla}^{2}\left(h_{\kappa} \circ d_{p}\right)=\left(\sin _{\kappa} \circ d_{p}\right) \cdot g
$$

If $\kappa$ is non-positive, $M$ admits a unique center of gravity. 


\section{Proof}

\subsection{Preliminaries}

We first prove proposition 1: the claim is trivial for $\kappa=0$. For non-zero $\kappa$, it is wellknown that if we equip the space $\mathbb{R}^{n+2}$ with the pseudo-metric $\langle x, y\rangle=\varepsilon x_{0} y_{0}+$ $\sum_{i=1}^{n+1} x_{i} y_{i}$ where $\varepsilon=\operatorname{sign} \kappa$ and its associated Levi-Civita pseudo-Riemannian connection $D$, then, a model space for $\mathbb{M}$ is the sphere $\mathbb{S}^{n+1}(1 / \sqrt{\kappa})=\{x \in$ $\left.\mathbb{R}^{n+2} /\langle x, x\rangle=1 / \kappa\right\}$ for positive $\kappa$ and the upper hyperboloid $\mathbb{H}^{n+1}(-1 / \sqrt{-\kappa})=$ $\left\{x \in \mathbb{R}^{n+2} /\langle x, x\rangle=1 / \kappa\right.$ and $\left.x_{0}>0\right\}$ for negative $\kappa$, with the induced metric, which is Riemannian. The announced formula follows then from two elementary facts: the distance of two points $x$ and $y$ of $\mathbb{M}$ is $d(x, y)=\sin _{\kappa}^{-1}(\kappa\langle x, y\rangle)$ which implies that $h_{\kappa} \circ d_{p}$ is the restriction to $\mathbb{M}$ of the linear form $-\langle p, \cdot\rangle+1 / \kappa$ on $\mathbb{R}^{n+2}$. On a second hand, $\left(D_{X} Y\right)_{q}=\left(\widetilde{\nabla}_{X} Y\right)_{q}-\kappa\langle X, Y\rangle q$ for any point $q \in \mathbb{M}$ and vector fields $X, Y$ on $\mathbb{M}$. We deduce immediatly that if $\gamma$ is a 1 -time speed geodesic of $\mathbb{M}$, then $(\mathcal{E} \circ \gamma) "(t)=\int_{M} \widetilde{\nabla}^{2}\left(h_{\kappa} \circ d_{q}\right)_{\gamma(t)}\langle\dot{\gamma}(t), \dot{\gamma}(t)\rangle \cdot d \nu(q)=\int_{M}\left(\sin _{\kappa} \circ d_{q}\right)(\gamma(t)) \cdot d \nu(q)>$ 0 , which shows the strict convexity of $\mathcal{E}$ and proves the assertion.

As $c$ is a critical point of $\mathcal{E}$, we note that $0=\langle\widetilde{\nabla} \mathcal{E}(c), u\rangle=\int_{M}\left\langle\widetilde{\nabla}\left(h_{\kappa} \circ d_{q}\right)(c), u\right\rangle$. $d \nu(q)$ for any $u \in U_{c} \mathbb{M}$ and the key point is the following:

Lemma. Let $u$ and $v$ be unit vectors tangent to $\mathbb{M}$ at $c$ and $q$ respectively, $q$ being arbitrary in $\mathbb{M}$. Then the smooth function $F_{c, u}: \mathbb{M} \rightarrow \mathbb{R}: q \mapsto\left\langle\widetilde{\nabla}\left(h_{\kappa} \circ d_{q}\right)(c), u\right\rangle$ satisfies:

$$
\begin{aligned}
\int_{U_{c} \mathbb{M}} F_{c, u}^{2}(q) \cdot d u & =\omega_{n+1} \cdot \operatorname{sn}_{\kappa}^{2}\left(d_{c}(q)\right) \\
\int_{U_{c} \mathbb{M}}\left\langle\widetilde{\nabla} F_{c, u}(q), v\right\rangle^{2} \cdot d u & =\omega_{n+1}\left\{1-\left\langle v, \widetilde{\nabla} d_{c}(q)\right\rangle^{2} \kappa \operatorname{sn}_{\kappa}^{2}\left(d_{c}(q)\right)\right\}
\end{aligned}
$$

where $d u$ is the canonical measure of $U_{c} \mathbb{M}$ and $\omega_{n+1}$ the volume of the unit ball of $\mathbb{R}^{n+1}$.

Formula (5) follows from the classical trick on quadratic forms:

Proposition 2. Let $\mathfrak{B}: V \times V \rightarrow \mathbb{R}$ be a bilinear form on a real $(n+1)$-dimensional inner product space $V$ and $(S, d u)$ the unit sphere of $V$ endowed with its canonical measure du. Then

$$
\int_{S} \mathfrak{B}(u, u) \cdot d u=\omega_{n+1} \cdot \operatorname{trace} \mathfrak{B}
$$

To prove (6), we introduce for convenience the function $\mu_{\kappa}(t)=\operatorname{sn}_{\kappa} t / t$ and we set 
$\ell=d(c, q)$. Then we can write $F_{c, u}(q)=-\mu_{\kappa}(\ell)\left\langle\exp _{c}^{-1} q, u\right\rangle$ and

$$
\left\langle\widetilde{\nabla} F_{c, u}(q), v\right\rangle=-\dot{\mu}_{\kappa}(\ell)\left\langle\widetilde{\nabla} d_{c}(q), v\right\rangle\left\langle\exp _{c}^{-1} q, u\right\rangle-\mu_{\kappa}(\ell)\langle\widetilde{\nabla} J(0), u\rangle
$$

where $J:[0,1] \rightarrow T \mathbb{M}$ is the unique Jacobi vector field along the geodesic $\gamma:$ $[0,1] \rightarrow \mathbb{M}: t \mapsto \exp _{c}\left(t \exp _{c}^{-1} q\right)$ in $\mathbb{M}$ joining $c$ to $q$ with the initial conditions $(J(0), J(1))=(0, v)$. Let us write $v=v^{T}+v^{\perp}$ where $v^{T}=\left\langle v, \widetilde{\nabla} d_{c}(q)\right\rangle \widetilde{\nabla} d_{c}(q)=$ $\langle v, \dot{\gamma}(1)\rangle \dot{\gamma}(1) / \ell^{2}$ is the tangential component of $v$ relatively to the geodesic speed vector $\dot{\gamma}(1)$. Solving the differential equation satisfied by $J$, one easily obtains that

$$
J(t)=\frac{\left\langle v, \widetilde{\nabla} d_{c}(q)\right\rangle}{\ell} t \dot{\gamma}(t)+\frac{\operatorname{sn}_{\kappa}(t \ell)}{\operatorname{sn}_{\kappa} \ell} P^{\perp}(t)
$$

where $P^{\perp}$ is the unique parallel vector field along $\gamma$ with $P^{\perp}(1)=v^{\perp}$. This implies that

$$
\widetilde{\nabla} J(0)=\frac{\left\langle v, \widetilde{\nabla} d_{c}(q)\right\rangle}{\ell} \dot{\gamma}(0)+\frac{1}{\mu_{\kappa}(\ell)} P^{\perp}(0)
$$

So,

$$
\begin{aligned}
\left\langle\widetilde{\nabla} F_{c, u}(q), v\right\rangle & =-\left\langle\left\{\dot{\mu}_{\kappa}(\ell)+\frac{\mu_{\kappa}(\ell)}{\ell}\right\}\left\langle v, \widetilde{\nabla} d_{c}(q)\right\rangle \dot{\gamma}(0)+P^{\perp}(0), u\right\rangle \\
& =-\left\langle\frac{\sin _{\kappa} \ell}{\ell}\left\langle v, \widetilde{\nabla} d_{c}(q)\right\rangle \dot{\gamma}(0)+P^{\perp}(0), u\right\rangle
\end{aligned}
$$

As $P^{\perp}$ is parallel along the geodesic $\gamma$ and perpendicular to the geodesic at $t=$ $1, P^{\perp}$ is of constant norm $\left|v^{\perp}\right|$ and always perpendicular to the geodesic. By proposition 2 and the straightforward relation $\sin _{\kappa}^{2} t+\kappa \operatorname{sn}_{\kappa}^{2} t=1$, one obtains that

$$
\frac{1}{\omega_{n+1}} \int_{U_{c} \mathbb{M}}\left\langle\widetilde{\nabla} F_{c, u}(q), v\right\rangle^{2} \cdot d u=\left|v^{T}\right|^{2} \sin _{\kappa}^{2} \ell+\left|v^{\perp}\right|^{2}=1-\left|v^{T}\right|^{2} \kappa \operatorname{sn}_{\kappa}^{2} \ell
$$

\subsection{Proof of the theorem}

Let $\eta$ be the outward smooth unit vector field normal to $M$, that is the one pointing in the opposite direction to $D$. The previous lemma, the Green formula which by proposition 1 is: $\int_{M}\left\langle\widetilde{\nabla}\left(h_{\kappa} \circ d_{c}\right), \eta\right\rangle \cdot d \nu=\int_{\Omega} \widetilde{\Delta}\left(h_{\kappa} \circ d_{c}\right) \cdot d \tilde{\nu}=(n+1) \int_{\Omega} \sin _{\kappa} \circ d_{c} \cdot d \tilde{\nu}$ and the generalized Hsiung-Minkowski formulae [1]: $\int_{M}\left\{H_{i} \sin _{\kappa} \circ d_{c}+H_{i+1}\left\langle\widetilde{\nabla}\left(h_{\kappa} \circ\right.\right.\right.$ $\left.\left.\left.d_{c}\right), \eta\right\rangle\right\} \cdot d \nu=0$ for any $i \in \llbracket 0, n-1 \rrbracket$ show formulae $(2)$ and (3) as easy consequences of (1). Formulae (5) et (6) prove (1): indeed, if we denote by $f_{c, u}$ the restriction of $F_{c, u}$ to $M$, then $\left|\nabla f_{c, u}\right|^{2}=\left|\widetilde{\nabla} F_{c, u}\right|^{2}-\left\langle\widetilde{\nabla} F_{c, u}, \eta\right\rangle^{2}$ and we deduce immediatly from (6) that

$$
\frac{1}{\omega_{n+1}} \int_{U_{c} \mathbb{M}}\left|\nabla f_{c, u}\right|^{2} \cdot d u=n-\left(1-\left\langle\eta, \widetilde{\nabla} d_{c}\right\rangle^{2}\right) \kappa \mathrm{sn}_{\kappa}^{2} \circ d_{c}
$$


The definition of $c$ imply that the integrals of the functions $\left(f_{c, u}\right)_{u \in U_{c} \mathbb{M}}$ on $M$ all vanish. By the Rayleigh min-max principle, the first positive Laplacian eigenvalue of $M$ satisfy $\lambda_{1} \int_{M} f_{c, u}^{2} \cdot d \nu \leq \int_{M}\left|\nabla f_{c, u}\right|^{2} \cdot d \nu$ for any vector $u \in U_{c} \mathbb{M}$ and equality is achieved if and only if $f_{c, u}$ is an eigenfunction, that is $\Delta f_{c, u}=\lambda_{1} f_{c, u}$. Integrating this last inequality on $U_{c} \mathbb{M}$, using Fubini theorem, (5) and (7), we obtain

$$
\lambda_{1} \int_{M} \operatorname{sn}_{\kappa}^{2} \circ d_{c} \cdot d \nu \leq \int_{M}\left\{n-\left(1-\left\langle\eta, \widetilde{\nabla} d_{c}\right\rangle^{2}\right) \kappa \mathrm{sn}_{\kappa}^{2} \circ d_{c}\right\} \cdot d \nu
$$

If $\kappa$ is negative, inequality (4) is immediate. For non-negative $\kappa$, the right term is bounded from above by $n \mathrm{Vol} M$ and we arrive to the announced inequality (1).

If $\kappa$ is zero and equality holds, then $\Delta f_{c, u}=\lambda_{1} f_{c, u}$ for any vector $u$. In order to be self-contained, we recall the classical argument [4] which concludes the proof: it is an easy result that $\Delta f_{c, u}=n H_{1}\langle\eta, u\rangle$ where $H_{1}$ is the 1-mean curvature of $M$. This implies that $n H_{1} \eta=-\lambda_{1} \widetilde{\nabla}\left(h_{\kappa} \circ d_{c}\right)$. We choose a local orthonormal basis $\left(X_{i}\right)_{1 \leq i \leq n}$ of principal vectors in $T M$ associated to the principal curvatures $\left(k_{i}\right)_{1 \leq i \leq n}$ and we differentiate this last equation by $X_{i}$. Using Weingarten equation and proposition 1 , we obtain $n\left(X_{i} H_{1}\right) \eta-n H_{1} k_{i} X_{i}=-\lambda_{1} X_{i}$. Taking its scalar product with $X_{i}$ and summing the relations obtained for all $i$, we deduce that $\lambda_{1}=n H_{1}^{2}$. This shows that $M$ is of constant 1 -mean curvature and by this way, included in a geodesic sphere centered at $c$ with radius $\sqrt{n / \lambda_{1}}$. As this geodesic sphere is also $n$-dimensional and connected, we deduce by the compactness of $M$ that $M$ coincides with this geodesic sphere.

If $\kappa$ is positive and equality holds, this means that $\eta= \pm \widetilde{\nabla} d_{c}$. Therefore, the gradient of $\left(h_{\kappa} \circ d_{c}\right)_{\mid M}$ satisfy $\left\langle\nabla\left(\left(h_{\kappa} \circ d_{c}\right)_{\mid M}\right), \xi\right\rangle=\left\langle\widetilde{\nabla}\left(h_{\kappa} \circ d_{c}\right), \xi\right\rangle=\left(\operatorname{sn}_{\kappa} \circ d_{c}\right)\left\langle\widetilde{\nabla} d_{c}, \xi\right\rangle=0$ for any vector field $\xi$ on $M$. As $M$ is connected, the function $h_{\kappa} \circ d_{c}$ is then constant on $M$, i.e. $M$ is included in a geodesic sphere centered at $c$. As this geodesic sphere is also $n$-dimensional and connected, we deduce by the compactness of $M$ that $M$ coincides with this geodesic sphere.

For negative $\kappa$, we note at last that equality cannot hold in (4): indeed, equality would imply that $\left\langle\eta, \widetilde{\nabla} d_{c}\right\rangle=0$ on $M$ and this is in contradiction with the first generalized Hsiung-Minkowski formulae which says that $0=\int_{M}\left\{\sin _{\kappa} \circ d_{c}+H_{1}\left\langle\widetilde{\nabla}\left(h_{\kappa} \circ\right.\right.\right.$ $\left.\left.\left.d_{c}\right), \eta\right\rangle\right\} \cdot d \nu=\int_{M}\left\{\sin _{\kappa} \circ d_{c}+H_{1} \cdot \operatorname{sn}_{\kappa} \circ d_{c}\left\langle\widetilde{\nabla} d_{c}, \eta\right\rangle\right\} \cdot d \nu$.

\section{Acknowledgements}

The author gratefully acknowledges many helpful conversations from Lamiae Jabri and Lucas Zakaria. This paper is dedicated to them. 


\section{References}

[1] Bivens, I., Integral formulas and hyperspheres in a simply connected space form, Trans. AMS 88 (1983), 113-118.

[2] Bleecker, D., Weiner, J., Extrinsic bounds on $\lambda_{1}$ of $\Delta$ on a compact manifold, Comment. Math. Helv. 51 (1971), 601-609.

[3] Reilly, R.C., On the first eigenvalue of the Laplacian for compact submanifolds of Euclidean space, Comment. Math. Helv. 52 (1977), 525-533.

[4] Takahashi, T., Minimal immersions of Riemannian manifolds, J. Math. Soc. Japan 18 (1966), 380-385.

Alain R. Veeravalli

Département de Mathématiques

Université d'Evry-Val d'Essonne

Boulevard des Coquibus

F-91025 Evry cedex

France

e-mail: Alain.Veeravalli@maths.univ-evry.fr

(Received: May 22, 2000) 